\title{
EL CUERPO Y LA CIUDAD. \\ UN ESTUDIO DE CASO DE ARTES PERFORMATIVAS COLABORATIVAS EN EL ESPACIO URBANO
}

The body and the city. A case study

of collaborative performing arts in the urban space

\begin{abstract}
Sofía Fernández Álvarez
Asistente de coordinación de exposiciones temporales en el Museo Nacional Centro de Arte Reina Sofía (MNCARs), Madrid, España.
\end{abstract}

RESUMEN: A través de la presentación de Body meets city, una experiencia de caminata colectiva y performance en el espacio público llevada a cabo en Nueva York en 2015, este estudio reflexiona acerca de las posibilidades y herramientas que tiene la práctica artística para definir, cuestionar y repensar las relaciones entre el cuerpo y el entorno urbano. El proyecto consta de una fase inicial de investigación sobre el terreno, así como de contacto y discusión con artistas y otros agentes, y una segunda en la que culmina finalmente en un evento de arte público. Partiendo de una base relativamente amplia de experiencias similares desarrolladas en distintos lugares del mundo, y de las propuestas teóricas de autores como Lefebvre o Careri, Body meets city enfatiza fundamentalmente el carácter artístico y colaborativo de las acciones que lo integran, así como la importancia del contexto local en el que éstas se desarrollan.

Palabras clave: arte público, artes performativas, prácticas colaborativas, espacio urbano. 
RESUM: A través de la presentació de Body meets city, una experiència de caminada col·lectiva i actuació en l'espai públic duta a terme a Nova York el 2015, aquest estudi reflexiona sobre les possibilitats i eines que té la pràctica artística per a definir, qüestionar i repensar les relacions entre el cos i l'entorn urbà. El projecte consta d'una fase inicial d'investigació sobre el terreny, així com de contacte i discussió amb artistes i altres agents, i una segona en la qual culmina finalment en un esdeveniment d'art públic. Partint d'una base relativament àmplia d'experiències semblants desenvolupades en distints llocs del món, i de les propostes teòriques d'autors com ara Lefebvre o Careri, Body meets city emfatitza fonamentalment el caràcter artístic i col-laboratiu de les accions que l'integren, així com la importància del context local en el qual aquestes es desenvolupen.

Paraules Clau: art públic, arts performatives, pràctiques col·laboratives, espai urbà.

ABSTRACT: Through the presentation of Body meets city, a collective walking and performance experience in the public space staged in New York in 2015, this study reflects on the possibilities and tools available to artistic practice to determine, question and re-think the relationships between the body and the urban environment. The first phase of the project involved local research, contact and discussion with artists and other agents, and the second phase culminated in a public art event. Starting from a relatively large base of similar experiences staged in different parts of the world, and from the theoretical proposals of authors such as Lefebvre or Careri, Body meets city essentially highlights the artistic and collaborative nature of its component actions, together with the importance of the local context in which they are enacted.

KEYWORDS: public art, performative arts, collaborative practices, urban space. 
$\mathrm{E}^{1}$ acto de caminar está siendo, recientemente, muy reivindicado como práctica artística, así como herramienta política o de transformación social. Los motivos a los que puede deberse este resurgimiento son de muy diversa índole: desde la recuperación del legado de la Internacional Situacionista (Wark, 2008) durante las últimas décadas hasta la herencia de la estética relacional de los años 90 (Bourriaud, 2008), que, sin embargo, se confronta con la fuerte repolitización del arte a la que hemos asistido durante la última década. Dados estos ingredientes, parece que la caminata colectiva, al proponer una experiencia social y estar al mismo tiempo cargada de contenido político, responde bastante bien a las circunstancias. También se podrían tener en consideración, como causas de esta emergencia, la coyuntura económica actual de Occidente, proclive a favorecer la creación no objetual, y el protagonismo que han tomado durante los últimos años, por motivos socio-políticos, las marchas y ocupaciones del espacio público. Así es como surgen prácticas performativas cargadas de contenido político basadas en el acto de caminar, que habitualmente suelen situarse a caballo entre el arte y el activismo, entre lo institucional y las iniciativas ciudadanas. En ellas se puede encontrar siempre, en mayor o menor medida, el poso teórico de Henri Lefebvre y sus ideas acerca de la producción y apropiación del espacio, desde su perspectiva marxista (Lefebvre, 1968-1969). Entre ellas podemos encontrar, por ejemplo, el Osservatorio Nomade creado por el colectivo italiano Stalker, la Revista Caminada que desarrolla en Madrid Hilario Álvarez desde hace años, o los ya clásicos Jane's Walks, que actualmente se desarrollan en todo el mundo y cuentan con un gran éxito de participación.

Evidentemente, el caminar per se carece de contenido suficiente como para ser considerado una herramienta política o una acción artística, de modo que la labor de dar un sentido determinado, una carga teórica, viene a ser tanto o más necesaria que en otro tipo de prácticas. En ese punto es donde se revela el papel del comisario, si bien éste se plantea de una forma relativamente heterodoxa en este tipo de prácticas sin objetos y fuera del espacio expositivo tradicional (lo que últimamente se viene denominando comisariado expandido). El comisariado aparece aquí como una labor consistente en dotar de un contenido sólido a un gesto cotidiano, combinar la marcha con otro tipo de acciones y también generar un evento o acontecimiento, más allá de la coordinación y la gestión cultural: localizar y poner en contacto a los 
artistas, generar un espacio de diálogo, crear un verdadero grupo en el que se aborde una reflexión conjunta; y por qué no, crear una red entre profesionales de intereses similares que pueda abrirles las puertas a diversas colaboraciones y proyectos futuros.

Éste es el contexto a partir del cual, durante el mes de agosto de 2015, se plantea Body meets city como proyecto de residencia curatorial en Residency Unlimited (Nueva York, EEUU). Aquí, el acto de caminar actúa como marco común e hilo conductor entre seis performances y acciones en el espacio público, cada una de las cuales pretende ser una propuesta distinta ante la cuestión de la relación entre el cuerpo y el espacio urbano. Dicha relación se puede dar a muchos niveles, y contemplarse desde muy diversas perspectivas: el recorrido físico del espacio como forma de (re)conocimiento del mismo, los flujos de viandantes y sus movimientos como una especie de coreografía inconsciente, el sonido como mediador entre la arquitectura y lo psicológico, las dinámicas sociales que se establecen dentro de un contexto urbano específico... Las acciones artísticas llevadas a cabo dentro de Body meets city exploran esta relación aportando miradas diversas, pero siempre atendiendo a dos condiciones básicas: establecer un diálogo con el lugar preciso en el que se inscriben y constituirse a través de la interacción con el público.

\section{Desarrollo del proyecto}

La primera parte de Body meets city como proyecto consistió en localizar y contactar a artistas que trabajaran, o hubieran trabajado en algún punto de sus carreras, sobre cuestiones como el cuerpo, el espacio urbano y la participación activa del público, y que se encontraran en la ciudad de Nueva York en aquel momento. Los participantes, finalmente, fueron: Juanli Carrión (español, residente en Nueva York), David Helbich (alemán, residente en Bruselas), Diana Policarpo (portuguesa, residente en Londres), John C. González (estadounidense, residente en Nueva York), Qinmin Liu (china, residente en San Francisco) e Ismael Kachtihi del Moral (franco-marroquí, residente en Reims). A partir del primer contacto, y manteniendo un diálogo abierto entre los propios artistas y con la comisaria, se fueron diseñando las seis acciones para el evento. Cuatro de ellas se concibieron específicamente para Body meets city, mientras que las dos restantes fueron adaptaciones de 
acciones llevadas a cabo previamente en otras ciudades o en otro tipo de espacios. En este segundo caso, se trataba de que Body meets city diera la posibilidad de transponer una misma acción de un contexto a otro diferente, explorando así las variaciones que el elemento local induce.

En una reunión previa al evento, se expusieron las propuestas de cada uno de los artistas y se escogió colectivamente la fecha y el lugar en el que llevar a cabo el recorrido con las acciones. Se decidió partir del comienzo de Fulton Street, en Brooklyn, y recorrer toda esta calle hasta descender a Lafayette Avenue a la altura de la Brooklyn Academy of Music (BAM). Se trata de una zona que recientemente ha experimentado rápidos cambios sociales, comerciales y arquitectónicos, y donde de hecho, aún se siguen produciendo. La calle Fulton y sus inmediaciones están pasando de ser un área periférica de la ciudad de Nueva York a incorporarse al centro, a medida que éste crece y se expande. En pleno proceso de cambio, actualmente conviven allí las tiendas de marcas internacionales - Fulton es ahora una de las principales calles comerciales de Brooklyn - con los puestos callejeros, los pisos antiguos con los apartamentos de lujo, las minorías étnicas de clase media-baja con los turistas. Estas condiciones de dinamismo, estado de tensión y transición en muchos aspectos, así como el gran flujo de viandantes y la heterogeneidad demográfica hicieron de ésta una zona interesante para llevar a cabo las acciones de Body meets city. A través de la red, y con la colaboración de Residency Unlimited y Spain Culture New York, se creó un evento abierto al público para el cual el grupo se daba cita en un punto y hora determinados para iniciar el recorrido a pie.

La primera acción del recorrido fue ${ }_{N Y C M K}$, de Juanli Carrión, que tuvo lugar al principio de la calle Fulton, un tramo peatonal. En esta acción, el artista extendió en el suelo, a modo de puesto callejero, varias camisetas con el conocido logotipo «I love New York», tres sprays de los colores primarios de impresión (negro, magenta, cian y amarillo) y sus respectivos stencils de papel, cada uno de ellos con la letra inicial de su color correspondiente (Y, C, M y K). Carrión ofrecía a los viandantes camisetas gratis, a cambio de que le dijeran cuál de los cuatro colores primarios identificaban con la ciudad de Nueva York. Cuando la persona elegía, el artista intervenía la camiseta con un 
stencil de la primera letra del color, la entregaba a su nuevo propietario y le pedía hacerle una foto con ella puesta, a modo de documentación.

Con el incremento de los precios en el barrio, los comercios multinacionales y franquiciados se imponen poco a poco, mientras que los locales a duras penas resisten, dando sus últimos coletazos. Entre ellos, los puestos callejeros que salpican las aceras de Fulton, modelo del cual Juanli Carrión se apropia de forma efímera, en un gesto de reivindicación y apoyo. Con esta acción, el artista subvierte la lógica de intercambio de mercancía por dinero, que es la imperante en una zona comercial como ésta, y convierte la transacción en un regalo a cambio de una reflexión. Por otra parte, la pregunta acerca del color con el que los transeúntes identifican la ciudad de Nueva York explora los valores y conceptos inconscientemente asociados a los colores: cuestiones de género, de raza y cultura, interesantes de explorar en un entorno urbano de población tan heterogénea como ésta.

La siguiente acción fue la del artista David Hebich, titulada Fulton Track en continuidad con otros Tracks que lleva realizando varios años en distintas ciudades (Bruselas, Riga, Kortrijk, Bergen y Maastricht). Su pieza consistió en hacer que el público le siguiese, en silencio y en fila india, a través de unos grandes almacenes, para terminar en un garaje. Una vez allí, en una parte del garaje que se abría a la calle, se colocó frente al público para dirigir una serie de ejercicios de escucha del sonido ambiente y percepción del espacio a través de la acústica.

La pieza de Helbich, al igual que su trabajo en general, tiene como base fundamental la activación del público, que no puede percibir la obra a menos que la realice, en lo que el artista suele denominar auto-performatividad (self-performativity). En la primera parte de Fulton Track, se ponía en cuestión la gestualidad habitual del desplazamiento de las personas en un espacio comercial. La fila india, el silencio, los recorridos aparentemente sin sentido entre los expositores de los grandes almacenes y los cambios de ritmo que el artista marcaba al grupo causaban la extrañeza de la gente alrededor porque incumplían las leyes no escritas sobre el movimiento en un lugar así, o mejor dicho, las expectativas de quienes diseñan unos grandes almacenes induciendo a un recorrido del espacio determinado, que va encaminado, además a un fin muy concreto: el consumo. La segunda parte tenía más que ver con la 
auto-performatividad y la experiencia del espacio a través del sonido. Aunque habitualmente se asocie el reconocimiento del espacio circundante con su recorrido físico, lo acústico tiene también un papel importante, que Helbich recalca aquí con una serie de ejercicios de escucha y concentración selectiva.

Tras salir del garaje, el grupo se dirigió hacia la plaza Albee, en cuyo centro hay mesas y sillas disponibles para el uso público. En una de las mesas, la artista Diana Policarpo había colocado un altavoz al que el público se acercaba para escuchar. El sonido que el altavoz emitía era una composición urbana, grabada y editada por Policarpo, en la que se entremezclaban grabaciones de campo, percusiones hechas sobre el cuerpo de la propia artista, la voz de ella sugiriendo ejercicios de mirada y escucha hacia diversos elementos de la plaza y también testimonios orales de los vecinos de la zona, donde explicaban los cambios que había experimentado su barrio durante los últimos años.

Una vez más, la propuesta, titulada Making room, tiene que ver con la experiencia a través del sonido, pero ya no como relación física con el espacio, sino como transmisión de conocimiento acerca del lugar. El cuerpo de la artista no se encuentra presente en la pieza (ella está allí simplemente como un miembro más del público); su presencia se actualiza en diferido, a través de la voz y de la percusión sobre él, pregrabada. Lo que el público, reunido en torno a una mesa, va conociendo acerca del lugar en el que se encuentra, no es únicamente lo que le llega a través de los sentidos, sino también los datos históricos y psicogeográficos que la grabación le proporciona, como adición a la experiencia del lugar, de la plaza pública.

En la misma plaza que la acción anterior tuvo lugar la de John C. González, A collection of solitary exercises for a public space. El artista repartió tarjetas en las que se proponían diferentes ejercicios de percepción y experimentación del lugar, explotando lo imaginativo y huyendo de las experiencias convencionales. Las tarjetas invitaban a presionar el cuerpo contra ciertos elementos arquitectónicos, a esforzarse por ver en blanco y negro o resaltando ciertos colores, a adoptar poses de defensa contra una amenaza imaginaria... Ejercicios que requerían del público un esfuerzo por imponer su imaginación a su percepción sensorial, o por que la primera alterase la segunda. Al terminar cada persona el ejercicio que le había tocado en su tarjeta, se le invitaba a 
intercambiarla con la de otra persona, produciendo una interacción entre los participantes. Esta norma de funcionamiento, lo mismo que otras características de la acción propuesta por González, vinculan el hecho artístico al juego, desdibujando los límites entre uno y otro, definiendo a través del arte al viandante, al ciudadano, al público, como homo ludens (Huizinga, 1938-1999) en el siglo XXI.

Esta colección de ejercicios había sido previamente diseñada para el espacio de una galería, y de hecho, se había realizado, unos meses atrás, en la Mills Gallery de Boston. Para Body meets city, John C. González tuvo que modificar las propuestas, teniendo que reflexionar acerca de las diferencias entre el espacio privado cerrado y una plaza pública como Albee, donde el público, las dimensiones espaciales, los elementos visuales y auditivos, la luz, etc., son totalmente diferentes.

En The imitation of Mao's first PM campaign, la artista Qinmin Liu instó al público participante a que imitase sus posturas, deteniendo al grupo para posar en diversos puntos de la calle Fulton. También pidió, por favor y por escrito, que se le tomaran fotos. Las poses que Liu adoptaba - a menudo procedentes de la danza, a la que lleva dedicándose largos años - siempre iban acompañadas de la mirada fija hacia la pantalla de su teléfono móvil, y así es cómo el público debía también colocarse.

Con esta acción, la artista exploraba y ponía de relieve la gestualidad, tan codificada, de las personas que transitan el espacio público, siempre mirando la pantalla de sus dispositivos electrónicos, y a menudo también fotografiando - más que mirando y viendo directamente - aquello que les rodea. Al asociar esta gestualidad cotidiana con la danza, Liu propone una visión en la que aquélla es entendida como un tipo de coreografía: un conjunto de pasos y figuras corporales compartidas e inconscientemente coordinadas entre quienes comparten el espacio público. Al mismo tiempo, la artista inspira su acción en una leyenda que se suele contar a los niños en las escuelas de China, según la cual Mao Tse Tung solía practicar ejercicios de concentración yendo a tratar de leer a una bulliciosa zona de la ciudad de Changsha. Hoy en día, esos ejercicios los realizan cada día prácticamente todos los ciudadanos de los países desarrollados, al centrar su atención en una pequeña pantalla mientras se encuentran en medio del bullicio y la sobrecarga de estímulos de 
una gran ciudad, como ocurre en esta calle de Nueva York. La acción finalizó con la repentina huida de la artista en un taxi, que dejó al grupo perplejo y aplaudiendo, antes de continuar la marcha hacia el lugar donde tendría lugar la siguiente experiencia.

La última acción del recorrido a pie corrió a cargo de Ismael Kachtihi del Moral, quien convirtió en performance la construcción de una instalación urbana efímera. El artista colocó, en lo alto de una reja de la calle Lafayette, 160 barcos de papel que contenían el mensaje «Write me» junto a su dirección de e-mail. Los barcos quedarían allí para que los transeúntes pudieran cogerlos y llevarlos consigo, siendo invitados a escribir al artista.

La acción llevada a cabo en el contexto de Body meets city supone el inicio de un proyecto más amplio desarrollado por Kachtihi del Moral, en el que se problematiza acerca de la cuestión del viaje y la comunicación verbal. Basándose en su propia experiencia, el artista defiende la importancia de los vínculos afectivos que se establecen más allá de las palabras, por encima de la existencia de una lengua en común. Write me busca la correspondencia, inesperada, de personas anónimas que decidan establecer esa relación. Probablemente, el contacto se produzca en una lengua (el inglés) con la que el artista no está familiarizado, pero que, sin embargo, no impedirá la comunicación y el lazo afectivo, generado ya a partir de la acción artística y a través del objeto intermediario: el barco de papel. Con este punto de partida, como señalaba anteriormente, Kachtihi del Moral inicia un proyecto en el que va dejando sus barcos por muy diversas ciudades del mundo, y recibiendo por tanto correos electrónicos de una gran cantidad de personas anónimas que deciden responder a su proposición y tomar parte, sin saberlo, en su acción artística. La intención del artista es que este proyecto de arte relacional pueda ser también llevado al espacio expositivo en forma de instalación multimedia, en la que el texto de los e-mails recibidos interactúe con una videoproyección que evoca el tema del desplazamiento y el viaje, y en la que también tengan un papel protagonista cientos de barcos de papel que el público pueda llevarse, de modo que Write me se convierta en un proyecto siempre inconcluso, en un tipo de comunicación que, como cualquier otro, nunca termina, o nunca se sabe cuándo va a terminar. 


\section{Conclusiones}

La experiencia de Body meets city entronca con una serie de prácticas que son relativamente habituales actualmente, y que suelen llevar el nombre de walks o performative walks. Sin embargo, sustituye la clásica deriva situacionista (Debord, 1958), o el recorrido marcado por un guía, por el seguimiento del artista junto al cual, en cada momento, el grupo está realizando la acción artística, o por el recorrido hasta el punto donde tendrá lugar la siguiente. En función de su emplazamiento, del método por el que se involucra al público y la reflexión que proponen, las distintas acciones de Body meets city podrían etiquetarse como guided tours o activities, según la clasificación establecida por Kaprow para los happenings (Kaprow, 1967-2003).

El conjunto de las seis acciones propone un desplazamiento físico alternativo por la ciudad, en tanto que no responde a las direccionalidades y objetivos que el sistema político-económico hace imperar en la gran ciudad contemporánea: desplazarse entre la vivienda, el lugar de trabajo y los centros de ocio y consumo. Se trata de una propuesta, desde el campo del arte, de cuestionar y modificar las lógicas cotidianas de la vida urbana a través de la modificación del tipo de desplazamiento físico que se lleva a cabo en la ciudad. La práctica y la teoría artística se presentan así como herramientas críticas que trabajan con y sobre la vida cotidiana y real, más allá de los espacios restringidos, iniciados y a menudo herméticos en los que se suelen desplegar ambas, ante un público iniciado y limitado. Se trata, pues, de retomar de alguna manera la utopía vanguardista de poner el arte al servicio de la vida y de la actividad ciudadana, revisando sus planteamientos y posibilidades para adaptarlos al contexto presente.

Además de proponer, como ya se ha ido viendo anteriormente, seis experiencias distintas sobre la relación entre cuerpo y espacio público, las acciones llevadas a cabo en Body meets city también exponen seis métodos o estrategias para involucrar al público en la acción artística. Ofrecer un producto gratuito, caminar en un orden y a un ritmo determinado, poner en una plaza un altavoz que emite sonidos y palabras, hacer gestos y figuras no habituales en la calle $\mathrm{u}$ ofrecer libremente el dato de contacto de un artista son diferentes maneras de reclamar la atención del público transeúnte e incitarle a participar. Así, las personas que integran estas piezas performativas no son únicamente las que 
se dieron cita al inicio del recorrido, sino también otras que se van sumando a la marcha y a las acciones a lo largo del evento. El objetivo es dar lugar a un acontecimiento inusual en la calle; un encuentro en el que la construcción (colectiva $\mathrm{y}$, en cierto modo, lúdica) de una situación genere una reflexión conjunta acerca de la vida en la ciudad y la experiencia de transitarla.

\section{Referencias}

Bourriaud, N. (2008): Estética relacional, Adriana Hidalgo, Buenos Aires.

Debord, G. (1958): «Théorie de la dérive», Internationale Situationniste, nº 2, pp. 19-23.

Huizinga, J. (1999): Homo ludens, Madrid, Buenos Aires.

KAProw, A. (2003): «Pinpointing Happenings». En Kelley, J. (ed.), Essays on the Blurring of Art and Life, University of California Press, London.

Lefebvre, H. (1969): El derecho a la ciudad, Península, Barcelona.

WARK, M. (2008): 50 Years of Recuperation of the Situationist International, Princeton Architectural Press, New York. 
